\title{
WILEY-VCH
}

DOI: 10.1002/adts.201800054

Article type: Full Paper

\section{Mechanism and Regulation of Spontaneous Water Transport in Graphene-Based Nanoslits}

Yuying Wang * , Junbo Xu ${ }^{\#}$, Huajian Zhu, Steven Wang ${ }^{*}$, Chao Yang *

Y.Wang

CAS Key Laboratory of Green Process and Engineering, Institute of Process Engineering, Chinese Academy of Sciences, Beijing 100190, China

University of Chinese Academy of Sciences, Beijing 100049, China

Dr. Junbo Xu

CAS Key Laboratory of Green Process and Engineering, Institute of Process Engineering, Chinese Academy of Sciences, Beijing 100190, China

Huajian Zhu

College of Chemical Engineering, Nanjing Tech University, Nanjing 210009, China

Dr. Steven Wang

School of Engineering, Newcastle University, NE1 7RU, UK

Email: Steven.Wang@newcastle.ac.uk

Prof. Chao Yang

CAS Key Laboratory of Green Process and Engineering, Institute of Process Engineering, Chinese Academy of Sciences, Beijing 100190, China

University of Chinese Academy of Sciences, Beijing 100049, China

Email: chaoyang@ipe.ac.cn

Keywords: nanoslit; transport; molecular dynamics simulation; nano-fluidic diode; graphene

While nanometer-scale capillaries have long been theoretically and experimentally studied, their transport mechanisms have not yet been fully understood. For the first time, we theoretically clarify the flow control mechanism by Molecular Dynamics (MD) simulations of graphene-based nanoscale capillaries. We find the imbibition rate to comply with a modified Lukas-Washburn equation. It changes non-monotonically with the slit width due to a compromise in competition of density and slip length. Secondly, we find the relative scale of imbibition and evaporation rate is length-dependent. From short slits to long ones, the imbibition-evaporation process alters from evaporation controlled to imbibition controlled. 


\section{WILEY-VCH}

Finally, we employ hydrophilic modification to enhance the rates. External modification can spread the water and accelerate the evaporation. Within the slit, the flow is accelerated by a positive hydrophilic gradient but detained by a negative one. These findings can be applied in a novel nano-fluidic diode.

\section{Introduction}

Well-ordered nanoporous membranes hold the promise of achieving precise selectivity and high flux, ${ }^{[1]}$ which can directly benefit the fields of chemical separations, drug delivery and wastewater remediation. Modern fabricating technologies have taken advantage of some materials such as carbon nanotubes ${ }^{[2]}$ and graphene ${ }^{[3]}$ to realize well-ordered and precisely controlled membranes. Radha et al. ${ }^{[4]}$ employed graphene and its multilayers as archetypal materials to fabricate smooth nanoslits with selected widths through Van der Waals assembly. In addition, it can invariably transport water by the consistent progress of spontaneous imbibition and evaporation. For the potential in energy-saving and efficient liquid transport devices, curiosity has risen over the self-propelled fluid and the membranes that realize it.

Capillary imbibition is ubiquitous in nature as the sap rise in trees ${ }^{[5]}$ and in artificial domains like the lab-on-a-chip. ${ }^{[6]}$ It denotes to the spontaneous fluid flow pumped by the capillary force, i.e., the pressure drop across the liquid/vapor interface. The studies on pressure-driven nanoflows are fairly comprehensive, where only the resistance is channeldependent and we can decide which kind of channel to be more favorable. However, in capillary flows, both the driving force and resistance depend on the channel, including the channel size and surface properties. ${ }^{[7]}$ The Lukas-Washburn (L-W) equation ${ }^{[8]}$ was established on the classic hydrodynamics, which is widely used to describe the imbibition process. With the advances from microsystems to nanosystems, some modifications are made

to the L-W equation on account of the nano-confinement. For example, Henrich et al. ${ }^{[9]}$ proposed an extended L-W equation with lubrication treatment, which involves the slip length 


\section{WILEY-VCH}

of nanoflows. The dynamic contact angle is also argued to be essential in some theories. ${ }^{[10]}$ Despite those improvements, the imbibition of water is generally subject to a half-power law, i.e., $x \sim t^{1 / 2}$, where $x$ is the penetration height of water and $t$ denotes the time.

The evaporation is always neglected in macroscale applications because it is much slower than dynamic flow. In nano-devices, however, evaporation always receives more attention. In such devices, the thickness of fluid layers can sometimes reach nanoscale, which leads to a drastical increase in surface/volume ratio. And the evaporation will be more noticeable in comparison to the flow process. In addition, common enhancement like heating will be more efficient in nanoscale evaporation. ${ }^{[11]}$ All of these make the evaporation a more significant process in nano-scale than macro-scale. Other than the conventional factors like temperature, liquid surface area, air velocity and humidity etc., the interaction between liquid and solid also makes a difference in nano-devices. Stronger hydrophilicity may at some extent make the escape of liquid molecules difficult, but the spreading effect that comes with it is very advantageous to the evaporation. ${ }^{[12]}$

On the other hand, nanofluidics endows the solid surfaces with great powers to drive and control the fluid flow inside. Different from the macroscale devices, a slight change on the solid/fluid interaction can either promote or impede the fluidic process, and even can do both at the same time. The concept of the fluidic diode firstly referred to the ionic flow diodes, whose mechanism is quite similar to electronic diodes. ${ }^{[13]}$ But recently, researchers found it possible to control the simple fluids like water merely through surface modifications that based on hydrophilicity regulations or asymmetric structures. ${ }^{[14]}$ With anisotropic modifications and interactions, flow will provide anisotropic properties. In capillary flows, as the surface interactions act as the driving force as well as the resistance, the mechanism of a diode may be more complex.

In this work, we employ Molecular Dynamics (MD) simulation to study capillary imbibition and subsequent evaporation. Figure 1 shows the schemes of our simulation 


\section{WILEY-VCH}

systems. Our work provides theoretical proof of self-propelled fluids. In addition, surface modification is introduced, which can either boost the water transport, or block the water in specific direction. A capillary-based system to drive and control water can start from this work.

\section{Results}

\subsection{Water imbibition and the optimal slit size}

The imbibition process of water in our simulation is recorded as the increase of $x$ with $t$, and is displayed in Figure 2(a). Where $N$ is the number of removed graphene layers. A deceleration tendency with time can be found from the imbibition process in each slit. In addition, the imbibition overall slows down as the slit gets wider.

In a slit-like capillary specifically, if the surface slippage ${ }^{[15]}$ is taken into account, the L-W equation can be written as

$$
x=\sqrt{\left[\frac{(d+6 \lambda) \gamma \cos \theta}{3 \eta}\right] t}
$$

where $x$ is the penetration height within the capillary and $d$ is the width of the slit as shown in Figure 1(a). $\lambda$ refers to the apparent slip length characterized by the Navier slip condition, ${ }^{[16]} t$ denotes time, $\gamma$ and $\theta$ indicate the surface tension and the contact angle respectively, and $\eta$ is the viscosity.

Our simulation process of water imbibition in Figure 2(a) can be fitted by Equation 1, and one can attribute the deceleration in wider slits to the receding apparent slip length. We estimate the apparent slip length with surface tension and contact angle to be $65 \mathrm{mN} \cdot \mathrm{m}^{-1}$ and $60^{\circ}$ respectively according to previous studies, ${ }^{[17]}$ and with viscosity of $1 \times 10^{-10} \mathrm{~Pa} \cdot \mathrm{s}$ (bulk water viscosity) for all slits, ignoring the viscosity change of nano-confined water. ${ }^{[18]}$ The apparent slip length shown in Figure 2(b) is within the range of $0 \sim 5 \mathrm{~nm}$, conforming to the empirical prediction with contact angle. ${ }^{[19]}$ And its reducing tendency with an enlarging slit also aligns with previous reports. ${ }^{[20]}$ 


\section{WILEY-VCH}

For the sake of simplicity, we define $A$ as

$$
A=\frac{6 \eta}{(d+6 \lambda) \gamma \cos \theta}
$$

It has a measure unit of $\mathrm{s} \cdot \mathrm{m}^{-2}$. In the imbibition and evaporation series, when evaporation takes place, water impregnates to fill the slit again. To measure the replenishing rate, we define a terminal velocity $v_{\mathrm{T}}$ as the imbibition rate at the full length of the slit $L$. According to the L-W theory we employ, it can be given as

$$
v_{\mathrm{T}}=\frac{1}{A L}
$$

The terminal velocity is inversely proportional to the slit length. So in thinner membranes with shorter slits, when evaporation takes place, the slit can be quickly replenished by imbibing new water molecules. However, in thicker membranes, such replenishment can be slow. With the terminal velocity, we can calculate the terminal flux $Q$ of the unit membrane cross-sectional area:

$$
Q=\frac{v_{\mathrm{T}} \rho S}{S / \varepsilon}=v_{\mathrm{T}} \rho \varepsilon \quad\left(\mathrm{g} \cdot \mathrm{s}^{-1} \cdot \mathrm{nm}^{-2}\right)
$$

where $S$ is the cross-sectional area of the slit, and $\varepsilon$ is the porosity. As $v_{\mathrm{T}}$ is inversely proportional to $L$, we can multiply the terminal flux with $L$ for normalization. The normalized $Q^{*}$ is displayed in Figure 2(b). $Q^{*}$ shows a non-monotonic change with $N$, which shows the compromise in competition of the apparent slip length and the density. When we reduce $N$ and therefore reduce $d$ of the slits, the apparent slip length increases, as mentioned above, and the density decreases from the bulk level, which was also proved by previous works. ${ }^{[21]}$ The normalized flux is jointly determined by these two elements and has an optimal value when $d$ is around $2 \mathrm{~nm}$, which is in quantitative agreement with Ref.(4).

\subsection{Evaporation and the control step}

When normalized to a unified slit area, the evaporation rates for slits with different $N$ tend to be close to each other as shown in Figure 3. As the evaporation rate is independent of the 


\section{WILEY-VCH}

slit length, its $Q \sim L$ relation can be denoted by a horizontal line in Figure 3. The imbibition terminal rate, however, is reversely proportional to $L$ as indicated above by Equation 3. So one can see from Figure 3 that as $L$ enlarges from a nanometer to millimeter scale, $Q$ for imbibition reduces for almost one million times. The evaporation, often considered as a slow process, can therefore outpace the imbibition when the slit is long enough, namely $100 \mu \mathrm{m}$ in our simulation. As a consequence, in a self-propelled membrane with capillary imbibition and evaporation processes, neither of steps can be arbitrarily designated as a control step. As the slit length increases, the control step alters from evaporation to imbibition. In application, we hope the slits to be as short as possible, because the limiting rate will be relatively high, and the evaporation rate can be easily enhanced by exterior actions such as ventilation and absorption.

\subsection{Surface modification and process enhancement}

In light of the two situations shown in Figure 3, we propose targeting approaches to enhance the rate. For evaporation control, efforts have been made to make the water spreading on the external end face to increase its contact area with air. Specifically, we increase the hydrophilicity by grafting hydroxyl (-OH group) to the carbon sites. The hydroxyl sites induce the water molecules to spread on the external walls spontaneously as shown in Figure 1(b). As a result of the spreading, the surface area enlarges several times depending on the porosity of the membrane. In our single-slit systems with a porosity of 0.33 , the evaporation rate increases from the level of $10^{-16} \mathrm{~g} \cdot \mathrm{s}^{-1} \cdot \mathrm{nm}^{-2}$ to $10^{-15} \mathrm{~g} \cdot \mathrm{s}^{-1} \cdot \mathrm{nm}^{-2}$ as displayed in Figure 3.

We adopt the same modification to enhance the rate of the imbibition-controlled process, but in this case the modified sites are within the slits. In nanochannels with a high surface ratio, hydrophilicity enhancement can cause significant effects in the flux, ${ }^{[2]}$ especially when the flow is driven by surface tension. The modification has been applied to the internal surfaces in three patterns: thoroughly modified (100\% modified), upstream-50\% (U-50\%) 


\section{WILEY-VCH}

modified and downstream-50\% (D-50\%) modified as shown in Figure 1(c). From the MD simulation, as shown in Figure 4(a), we find that the imbibition is accelerated through modification in both the $100 \%$ and downstream-50\% modified slits. For the D-50\% case, one can find an evident acceleration in the second section. And for the $100 \%$ modified slit, the acceleration can be found from the very beginning of the imbibition process. However, the top-50\% modified slit holds no enhancement but detains the water at the junction. We find the water inside cannot cross the junction from the more hydrophilic surface to the less hydrophilic surface and it results in an impassable slit for water in this direction. Such a detaining effect is still valid when $N$ increases to 15 , the widest in this work. The snapshots in Figure 4(b) show the oscillation in water level at the hydrophilicity gradient. The huge resistance comes from the strong interaction between water and the more hydrophilic surface. By the two means we employed, the rates for both evaporation control and imbibition control can be accelerated. As shown in Figure 5, in a slit with $L=1 \mu \mathrm{m}$, the controlling rate of evaporation can be enhanced from $9 \times 10^{-16} \mathrm{~g} \cdot \mathrm{s}^{-1} \cdot \mathrm{nm}^{-2}$ to $4 \times 10^{-15} \mathrm{~g} \cdot \mathrm{s}^{-1} \cdot \mathrm{nm}^{-2}$. And in a slit with $L=100 \mu \mathrm{m}$, the controlling rate of imbibition enlarges from $4 \times 10^{-16} \mathrm{~g} \cdot \mathrm{s}^{-1} \cdot \mathrm{nm}^{-2}$ to $8 \times 10^{-16} \mathrm{~g} \cdot \mathrm{s}^{-}$ ${ }^{1} \cdot \mathrm{nm}^{-2}$ in a downstream-50\% modified slit and to $1 \times 10^{-14} \mathrm{~g} \cdot \mathrm{s}^{-1} \cdot \mathrm{nm}^{-2}$ in a $100 \%$ modified slit.

The imbibition process when the slit is in sectional surface properties can be interpreted theoretically as the L-W equation can be expanded. Like Equation 1, the equation for a sectional slit can be drawn:

$$
\begin{cases}x=\sqrt{\frac{2}{A_{1}}} \sqrt{t} \quad x \leq L_{1} & \\ x=L_{1}+\frac{-A_{1} L_{1}+\sqrt{\left(A_{1} L_{1}\right)^{2}+2 A_{2} t}}{A_{2}} & L_{1}<x \leq L_{2}\end{cases}
$$

where $L_{1}$ and $L_{2}$ represent the lengths of two slit sections. $A_{1}$ and $A_{2}$ are the resistance parameters of the two sections according to Equation 2. The water rise in the end-50\% modified slit with $N=5$ can be fitted with Equation 5 precisely as shown in Figure 4(a). For 


\section{WILEY-VCH}

the detaining effect we found in the U-50\% modified case, here we need to make an additional condition for Equation 5 that

$$
A_{1}>A_{2}>0
$$

When the terminal velocity is concerned, it can be given as

$$
v_{\mathrm{T}}=\frac{1}{A_{1} L_{1}+A_{2} L_{2}}
$$

And it can be further expanded to more sections as

$$
v_{\mathrm{T}}=\frac{1}{A_{1} L_{1}+A_{2} L_{2}+\cdots+A_{n} L_{n}}
$$

Similarly, $A_{1}>A_{2}>\ldots>A_{n}$ is required. The detailed derivations for all the equations in this section are presented in the supplementary materials.

Once a nanoslit has a gradient in hydrophilicity within it, and when there is no driving force other than the capillary force, it is traversable in the direction where the hydrophilicity gradually increases but not the other way around.

\section{Discussion}

As discussed above, a fluidic diode can be made out of the slit with differences in hydrophilicity inside. The flow is accelerated by a positive hydrophilic gradient but detained by a negative one. Similar diodes have been realized in some experiments. ${ }^{[23]}$ Nanofluidic diode with a half hydrophilic and half hydrophobic channel has already been proved valid, which can be easily interpreted by the capillary theory and surface energy. In a word, fluid will not occupy a hydrophobic surface spontaneously. But in this work, we relax the requirement, in which even when the whole slit is hydrophilic, a hydrophilicity gradient can still implement the diode. Hence, a further explanation is in demand.

The contact angle variation driven by edge effect has been previously studied. When water invaded on hydrophilic surface and came across with geometrical edge or wettability separation boundary, the pinning of the invading line would increase the contact angle to 


\section{WILEY-VCH}

minimize the interfacial free energy until it reached a critical angle, ${ }^{[24]}$ which is much larger than the intrinsic contact angle. When the edge effect occurs in a capillary problem, the increase in contact angle will lead to the diminution of driving force. And when the contact angle exceeds 90 degrees, the surface tension will be downward and no force can continuously drive the flow upwards. Therefore, the edge effect can be one reason for the water detaining from a more hydrophilic to a less hydrophilic section. To verify the theory, we calculate the free energy change. A bottom made of graphene is added to the original system, and by pushing which, one can propel the liquid even if the hydrophilicity difference prohibits it. Starting from the equilibrated state reached by the spontaneous flow as Figure 4(b) exhibits, we push the liquid within the slit rising for about $2 \mathrm{~nm}$ a time and then run enough time for equilibrium with the bottom graphene being fixed. In this way, we can increase the liquid level inside the slit without changing any other interaction of the system. Such process is repeated till the slit is filled with water. Above all, the liquid level stops at where it is every time the pushing force is repealed. No sustaining advance can be observed even if the hydrophilicity transition has already been passed. Besides, the total energy generated by pairwise interaction and its components, namely the energy from Van der Waals (VdW) interaction, and the coulomb force are recorded at each position as shown in Figure 6(a). All of them show a stepwise rise with the liquid level increasing above the transition. From the energy aspect, we prove that water cannot spontaneously flow from a more hydrophilic side to a less hydrophilic side by capillary force.

We consider the capillary flow as a flow driven by the surface tension caused by a curved surface formed within hydrophilic capillaries. The driving force will not vanish but only decrease when the water starts to contact the less hydrophilic half. There must be some resistant that stops the flow from advancing. Hence, it can be the anisotropic flow resistances in different directions that take effect. To test it, we make two slits with pure graphene and modified graphene separately, and both of them are filled with water. Minute force is applied 


\section{WILEY-VCH}

to the water inside those slits, and when the force rises from zero, the water is at first resting but then forms a constant velocity after a critical force and keeps increasing with the force. The force-velocity curves are displayed in Figure 6(b), which shows evident differences in the slit with/without modification. It means in the more hydrophilic slits, we need more drive to put the water in motion. As we know in the capillary cases, the drive is the surface tension which is stronger in more hydrophilic slits. Therefore, when water flows from the less hydrophilic half to the more hydrophilic half, it is the higher drive with a lower resistance. However, when water flows from the other way, it is the lower drive with a higher resistance, and then the flow is detained.

\section{Method}

Figure 1 shows the schemes of our simulation systems. In our simulation, a slit-like capillary is constructed by removing interlayers from the multilayered graphene ${ }^{[25]}$ as shown in Figure 1(a). We denote the width of the slit by the number $N$ of graphene layers that are removed. The explicit width $d$ can then be estimated as $N a$, where $a \approx 3.4 \AA$ is the effective thickness of one graphene layer. For its feasibilities in both fluidic and evaporating systems, ${ }^{[26]}$ the SPC/E model is used for water in this work. In the model, we have $\varepsilon_{\mathrm{OO}}=0.1553 \mathrm{kcal} \cdot \mathrm{mol}^{-1}$ and $\sigma_{\mathrm{OO}}=3.166 \AA$. The charge of oxygen and hydrogen are -0.820 and 0.410 electron charge respectively. The Ewald solver is used to calculate long-range interactions for charges. The carbon atoms are fixed neutral particles whose interaction with oxygen is modelled by the Lennard-Jones (L-J) potential with the parameters of $\varepsilon_{\mathrm{CO}}=0.0927 \mathrm{kcal} \cdot \mathrm{mol}^{-1}$ and $\sigma_{\mathrm{CO}}=3.283 \AA$ ${ }^{[4]}$. The cut-off diameter for the L-J interaction is $10 \AA$.

The system is constructed by multilayer graphene and a water reservoir in contact with it as shown in Figure 1(a). The figure has been cut to fit in and only the most important part is shown. The actual simulation box is $200 \times W \times 2 \mathrm{~nm}$, where $W$ depends on the slit width to ensure a unified porosity of 0.33 for each case. For example, when $N=5,5$ layers of graphene 


\section{WILEY-VCH}

are removed, and then another 5 layers are used as the walls on each side of the slit. Then $W=15 a=5.1 \mathrm{~nm}$. The $\mathrm{x}$-direction is the axial direction of the slit. The length of the slit is 24 $\mathrm{nm}$, and the reservoir has a deep of $150 \mathrm{~nm}$, which ensures the reservoir to be about 20 times as large as the slit. Therefore, the pressure change is negligible during the imbibition. The simulation box is periodic in all directions. But a graphene layer is set to prevent the molecules in reservoir flowing backwards. The system runs in NVT ensemble with a timestep of $1.0 \mathrm{fs}$, and the computation lasts for $2 \mathrm{~ns}$ for imbibition and $20 \mathrm{~ns}$ for evaporation.

When the slit is filled with water after the capillary imbibition, we continue the simulation to calculate the evaporation rate. Referring to Wang et al., ${ }^{[12]}$ when separate water molecules depart from the bulk water to a certain distance, $5 \mathrm{~nm}$ in this case, they are involved in an accelerating region and are collected at the top of the system as shown in Figure 1(b). The evaporation rate has been estimated via the accumulating rate of this collection.

Surface modification is applied respectively to the external surface (Figure 1(b)) and inner surface (Figure 1(c)) to accelerate the evaporation and imbibition. The modification is accomplished by grafting hydroxyl (-OH group) on the carbon of particular surface. $33.3 \%$ of the surface carbon sites are grafted with $-\mathrm{OH}$. The $-\mathrm{OH}$ group is hydrophilic as the contact angle with water before and after modification can be found in Figure 1(d).

All simulations are carried out in the Large-scale Atomic Molecular Massively Parallel Simulator (LAMMPS) software package.

\section{Conclusions}

In conclusion, $\mathrm{MD}$ simulation is employed to investigate capillary imbibition and evaporation. The imbibition rate is found to be influenced by a compromise in competition mechanism of density and slip length, reaching its maximum when slits are around $2 \mathrm{~nm}$ in width. When enlarging the slit length, the control step of the integrated imbibition-evaporation self-propelled system alters from evaporation to imbibition. The rate of each control step is 


\section{WILEY-VCH}

successfully accelerated by the surface modification of the hydrophilic groups. And a new sort of fluidic diode can be realized from a hydrophilic gradient inside the slit. It is worth noting that the hydrophilic modification has different effects for capillary flow and pressure-driven flow. In capillary flows, the hydrophilic modification prompts the flow by enhancing the surface tension. However, in pressure-driven flows, it hinders the flow for a stronger solid/liquid interaction, which we have clarified in our previous works. ${ }^{[27]}$

\section{Supporting Information}

Supporting Information is available from the Wiley Online Library or from the authors.

\section{Acknowledgements}

We thank Prof. Omar Matar and Prof. Richard M. Lueptow for their valuable comments on this work. Financial supports from the National Key R\&D Program of China (2016YFB0301701), National Natural Science Foundation of China (21490584, 91534105), Key Research Program of Frontier Sciences of CAS (QYZDJ-SSW-JSC030) and Jiangsu National Synergetic Innovation Center for Advanced Materials are gratefully acknowledged.

\# Yuying Wang and Junbo Xu contributed equally to this work.

Received: ((will be filled in by the editorial staff))

Revised: ((will be filled in by the editorial staff)) Published online: ((will be filled in by the editorial staff))

\section{References}

[1] a) X. Li, C-A. Fustin, N. Lefèvre, J-F. Gohy, S. D. Feyter, J. D. Baerdemaeker, W. Egger and I. F. J. Vankelecom, J. Mater. Chem. 2010, 20 (21), 4333-4339. b) M. Majumder, N. Chopra, R. Andrews and B. J. Hinds, Nature 2005, 438, 44.

[2] B. J. Hinds, N. Chopra, T. Rantell, R. Andrews, V. Gavalas and L. G. Bachas, Science 2004, 303, 62-65.

[3] a) B. Radha, A. Esfandiar, F. C. Wang, A. P. Rooney, K. Gopinadhan, A. Keerthi, A. Mishchenko, A. Janardanan, P. Blake, L. Fumagalli, M. Lozada-Hidalgo, S. Garaj, S. J. Haigh, I. V. Grigorieva, H. A. Wu and A. K. Geim, Nature 2016, 538, 222-225. b) S. Garaj, W. Hubbard, A. Reina, J. Kong, D. Branton and J. A. Golovchenko, Nature 2010, 467 (7312), 190-193.

[4] B. Radha, A. Esfandiar, F. C. Wang, A. P. Rooney, K. Gopinadhan, A. Keerthi, A. Mishchenko, A. Janardanan, P. Blake, L. Fumagalli, M. Lozada-Hidalgo, S. Garaj, S. J. Haigh, I. V. Grigorieva, H. A. Wu and A. K. Geim, Nature 2016, 538, 222-225. 


\section{WILEY-VCH}

[5] M. Denny, Eur. J. Phys. 2012, 33, 43-53.

[6] Oliver Geschke, Henning Klank and P. Telleman, Wiley-VHC: Germany, 2004.

[7] a) B. Henrich, C. Cupelli, M. Santer and M. Moseler, New J. Phys. 2008, 10 (11), 113022. b) C. Cupelli, B. Henrich, T. Glatzel, R. Zengerle, M. Moseler and M. Santer, New J. Phys. 2008, 10 (4), 043009.

[8] a) E. W. Washburn, Physical Review 1921, 17 (3), 273-283. b) B. V. Zhmud, F. Tiberg and K. Hallstensson, J. Colloid Interface Sci. 2000, 228 (2), 263-269.

[9] B. Henrich, C. Cupelli, M. Santer and M. Moseler, New J. Phys. 2008, 10 (11), 113022.

[10] C. Cupelli, B. Henrich, T. Glatzel, R. Zengerle, M. Moseler and M. Santer, New J. Phys. 2008, 10 (4), 043009.

[11] Y. Li, M. A. Alibakhshi, Y. Zhao, and C. Duan, Nano Lett. 2017, 17, 4813-4819.

[12] a) Y. Takata, S. Hidaka, J.M. Cao, T. Nakamura, H. Yamamoto, M. Masuda and T. Ito, Energy 2005, 30, 209-220. b) Shen Wang, Yusong Tu, Rongzheng Wan and H. Fang, $J$. Phys. Chem. B 2012, 116, 13863-13867.

[13] a) Rohit Karnik, Chuanhua Duan, K. Castelino, Hirofumi Daiguji and A. Majumdar, Nano Lett. 2007, 7 (3), 547-551. b) I. Vlassiouk and Z. S. Siwy, Nano Lett. 2007, 7 (3), 552-556.

[14] a) L. Li, J. Mo and Z. Li, Phys. Rev. Lett. 2015, 115, 134503. b) Zhenxing Wang, X. Yang, Zhongjun Cheng, Yuyan Liu, Lu Shao and L. Jiang, Mater. Horiz. 2017, 4, 701-708. c) Jingwen Mo, Long Li, JunWang and Z. Li, Phys. Rev. E 2016, 93, 033101.

[15] B. Ramos-Alvarado, S. Kuma and G. P. Peterson, Phys. Rev. E 2016, 93, 033117.

[16] J. Xu, C. Yang, Y.-J. Sheng and H.-K. Tsao, Soft Matter 2015, 11, 6916--6920.

[17] a) Jerzy Włoch, Artur P. Terzyk and P. Kowalczyk, Chem. Phys. Lett. 2017, 674, 98-102.

b) T. Werder, J. H. Walther, R. L. Jaffe, a. T. Halicioglu and P. Koumoutsakos, J. Phys. Chem. B 2003, 107 (6), 1345-1352.

[18] K. Ritos, D. Mattia, F. Calabrò and J. M. Reese, J. Chem. Phys. 2014, 140, 014702.

[19] a) D. M. Huang, C. Sendner, D. Horinek, R. R. Netz and L. Bocquet, Phys. Rev. Lett. 2008, 101 (22), 226101. b) L. Bocquet and E. Charlaix, Chem. Soc. Rev. 2010, 39, 10731079.

[20] a) Y. Wang, J. Xu, S. Wang and C. Yang, Nanoscale 2017, 9 (20), 6777-6782. b) E. Secchi, S. Marbach, A. Niguès, D. Stein, A. Siria and L. Bocquet, Nature 2016, 537, 210-213.

[21] A. Alexiadis and S. Kassinos, Chem. Eng. Sci. 2008, 63 (8), 2047-2056.

[22] G. Martic, F. Gentner, D. Seveno, D. Coulon and J. D. Coninck, Langmuir 2002, 18, 7971-7976.

[23] L. Wang, Y. Feng, Y. Zhou, M. Jia, G. Wang, W. Guo and L. Jiang, Chem. Sci. 2017, 8 (6), 4381.

[24] a) F-M. Chang, Y-J. Sheng and H-K. Tsao, Applied Physics Letters 2009, 95, 204107. b) F-M. Chang, S-J. Hong, Y.-J. Sheng and H-K. Tsao, J of Phys. Chem. C 2010, 114, $1615-1621$.

[25] a) B. Radha, A. Esfandiar, F. C. Wang, A. P. Rooney, K. Gopinadhan, A. Keerthi, A. Mishchenko, A. Janardanan, P. Blake, L. Fumagalli, M. Lozada-Hidalgo, S. Garaj, S. J. Haigh, I. V. Grigorieva, H. A. Wu and A. K. Geim, Nature 2016, 538, 222-225. b) C. Cheng, G. Jiang, C. J. Garvey, Y. Wang, G. P. Simon, J. Z. Liu and D. Li, Sci. Adv. 2016, 2, e1501272.

[26] a) M. A. González and J. L. F. Abascal, J Chem. Phys. 2010, 132 (096101). b) P. Varilly and D. Chandler, J Phys. Chem. B 2013, 117, 1419-1428. c) P. Mark and L. Nilsson, J Phys. Chem. A 2001, 105, 9954-9960.

[27] Y. Wang, J. Xu, S. Wang and C. Yang, Nanoscale 2017, 9 (20), 6777-6782. 
(a)

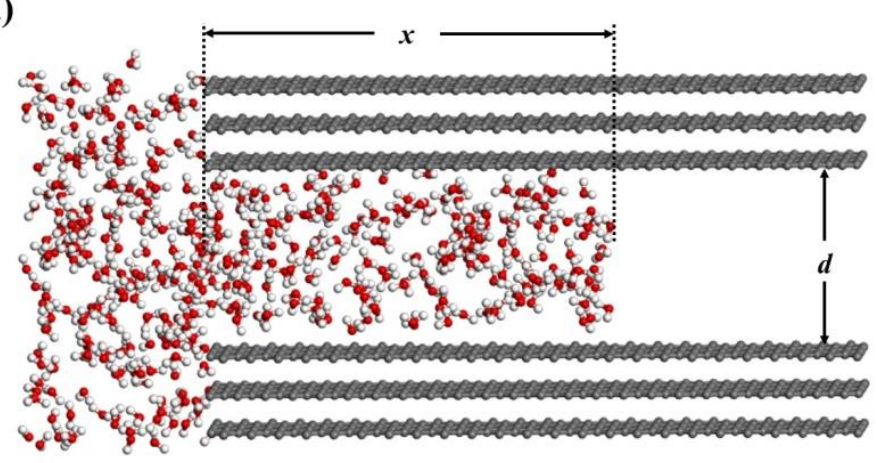

(b)

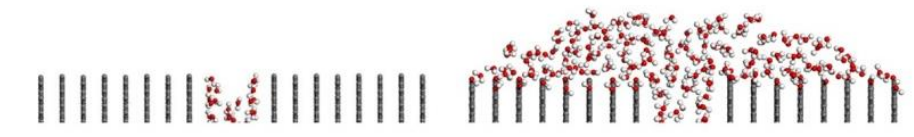

(c)
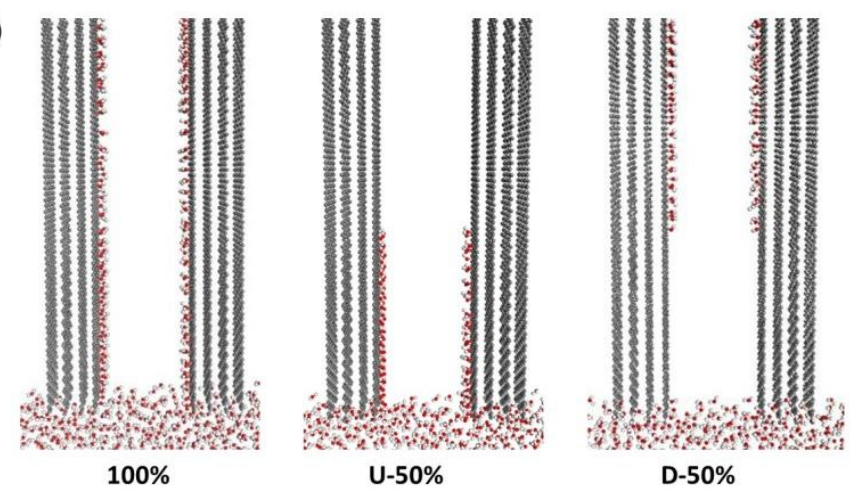

(d)

original

modified

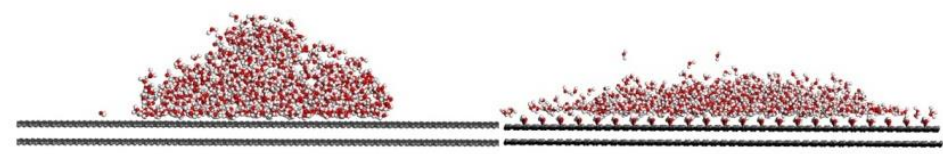

Figure 1. Partially enlarged details of schematic diagram: (a) for the whole system with reservoirs, the penetration length $\boldsymbol{x}$ measures the distance from the bottom of the slit to the water surface which is detected with an average local number density of $1 / 2$ bulk density, and $d$ is the width of slit; (b) for a local zoom at the slit exit, the scheme on the left denotes a regular system and the right one denotes a slit with external modification, which drive water to form a drop outside after filling the slit; (c) for the three different surface modifications, namely the thoroughly modified (100\% modified), upstream-50\% 


\section{WILEY-VCH}

(U-50\%) modified and downstream-50\% (D-50\%) modified; (d) for the contact angle on the graphene surface, $\theta \approx 60^{\circ}$ for original surface and $\theta \approx 15^{\circ}$ for modified surface. In these schematic graphs, we denote carbon in gray, oxygen in red and hydrogen in white. 

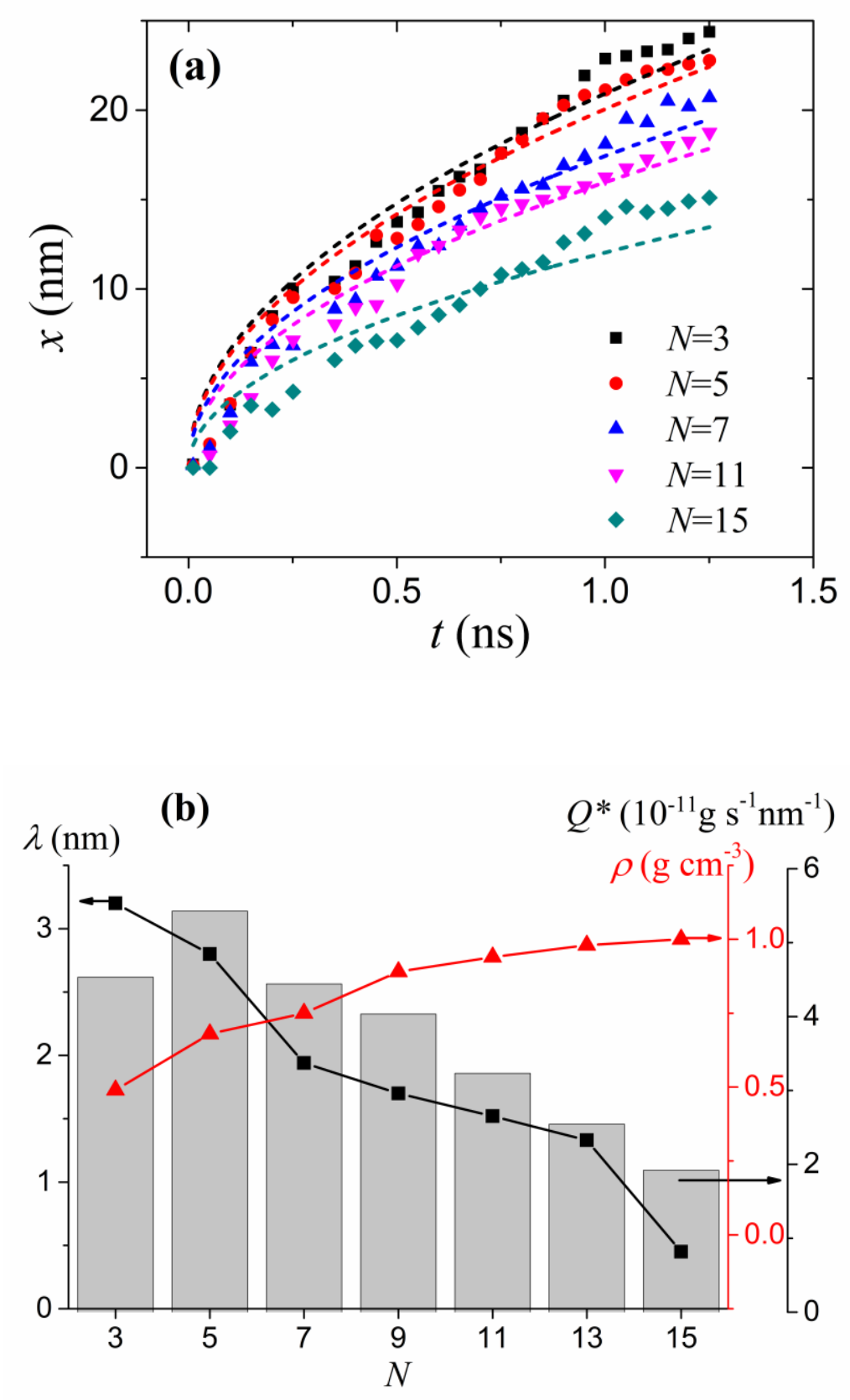

Figure 2. Capillary imbibition in slit-like nanochannels: (a) capillary rise with time during the imbibition process and its fitting of Eq.(1); (b) variation of normalized flux (bars), apparent slip length (squares), and density (triangles) with slit width represented by the number of graphene layers, $N$. 


\section{WILEY-VCH}



Figure 3. Imbibition and evaporation rates with various capillary lengths. 


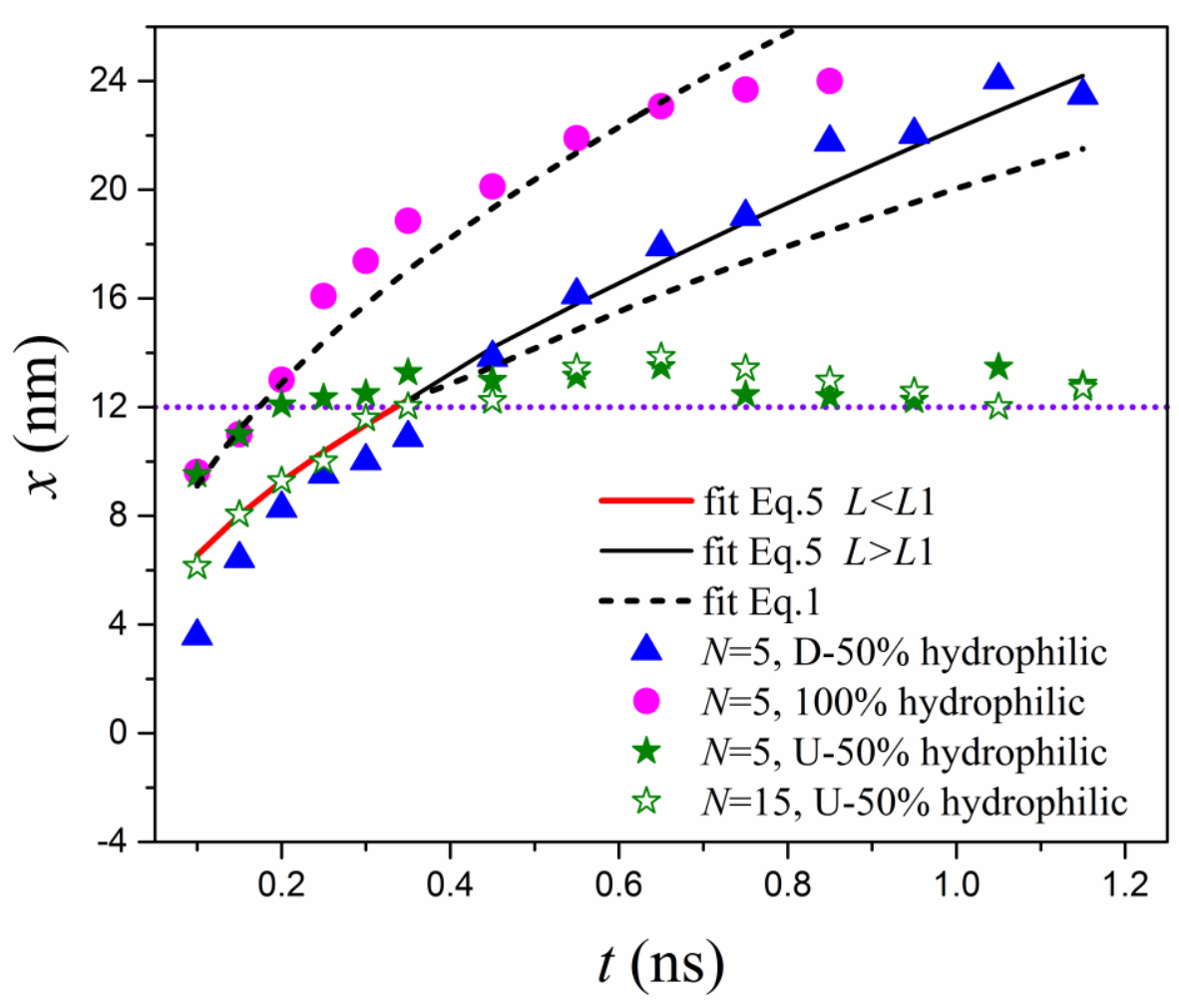

(a)

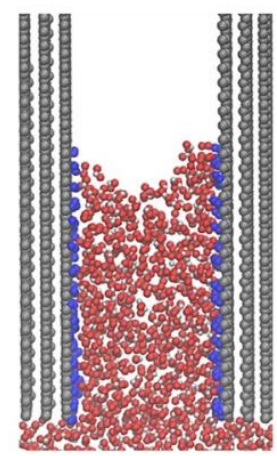

$t=0.2 \mathrm{~ns}$



$t=0.4 \mathrm{~ns}$

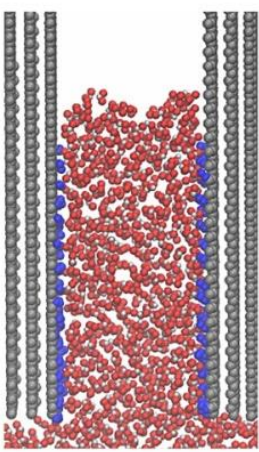

$t=0.6 \mathrm{~ns}$

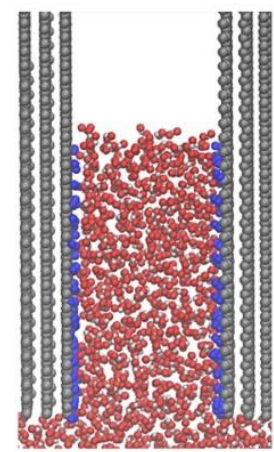

$t=0.8 \mathrm{~ns}$

(b)

Figure 4. Consequences of internal modification on slits: (a) flow rise in a half-length modified slit; (b) snapshot of water detention in a U-50\% modified slit where blue points are the grafted -OH group. 


\section{WILEY-VCH}



Figure 5. Integral flux of the imbibition-evaporation process, there are full and no external modification for the evaporation control, and there are full, half (D-50\%) and no internal modification for the imbibition control. 


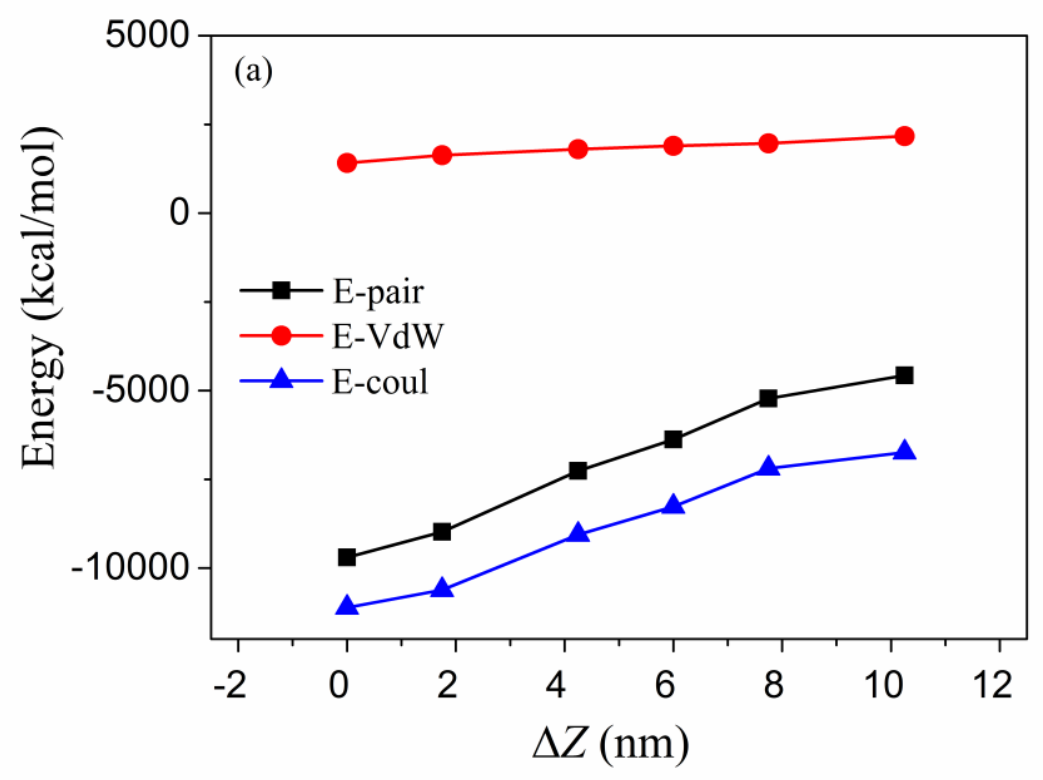

(a)

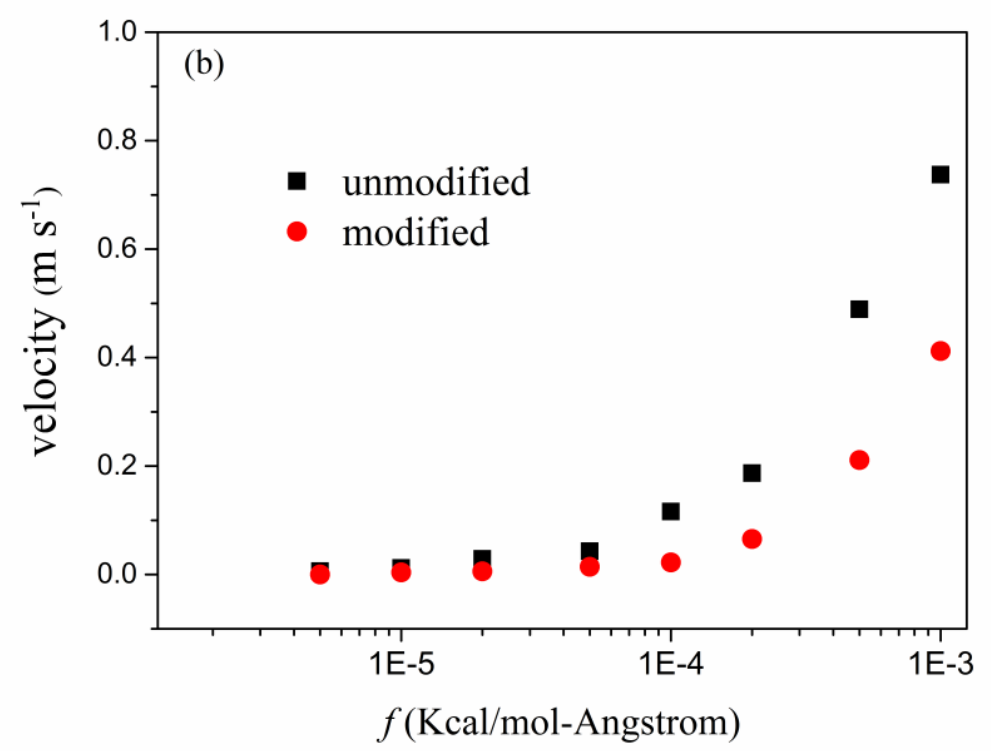

(b)

Figure 6. Analysis for the mechanism of fluidic diode: (a) System energy with liquid level higher than the transition, $\Delta Z$ is the distance between the water surface inside slits and the hydrophilicity transition place. Epair is the total energy generated from pairwise interactions; VdW and coul are respectively the energy generated from Van de Waals and coulomb forces. Where Epair=VdW+coul; (b) Driving force and velocity response in modified and unmodified slits. 


\section{WILEY-VCH}

\section{Table of Contents}

While it is widely accepted that nano-scale capillaries hold great potential in numerous applications, previous attempts to explain its discrepancies with micro-scale capillaries have failed. For the first time, we theoretically clarify the flow control mechanism of graphenebased nanoscale capillaries. Molecular simulations reveal that the imbibition rate of water in nanoslit is influenced by compromise-in-competition of confined density and slippage. We present a novel nanofluidic diode, where the flow can be precisely regulated by surface structure modification. This concept appears to be practically relevant in a number of nanofluidic systems.

Keywords: nanoslit; transport; molecular dynamics simulation; nano-fluidic diode; graphene

Yuying Wang \#, Junbo Xu ${ }^{\#}$, Huajian Zhu, Steven Wang ${ }^{*}$, Chao Yang *

Mechanism and Regulation of Spontaneous Water Transport in Graphene-Based Nanoslits

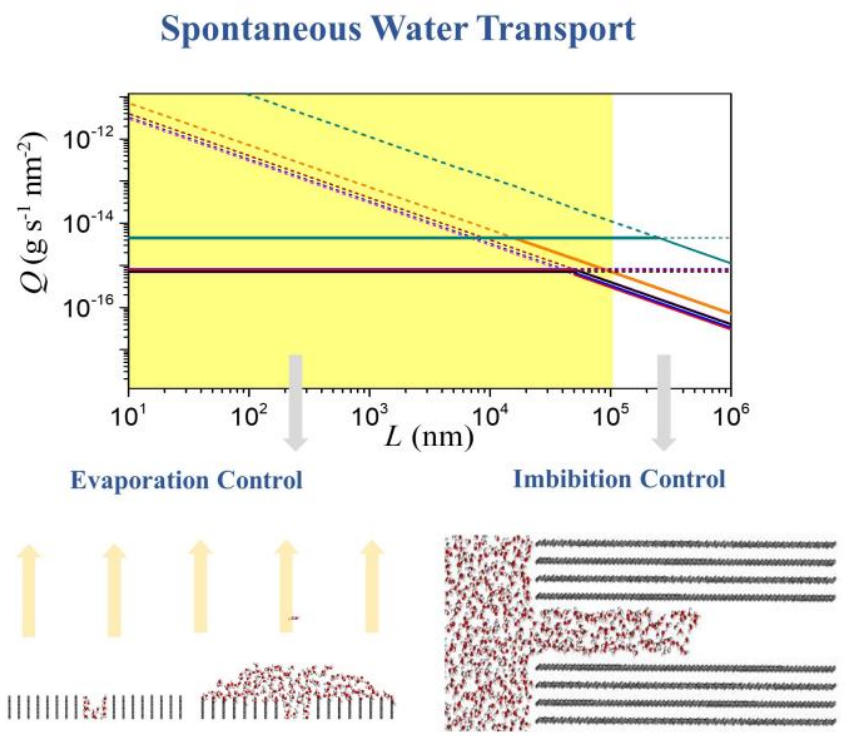




\section{WILEY-VCH}

Copyright WILEY-VCH Verlag GmbH \& Co. KGaA, 69469 Weinheim, Germany, 2016.

\section{Supporting Information}

\section{Mechanism and Regulation of Spontaneous Water Transport in Graphene-Based Nanoslits}

Yuying Wang\# , Junbo Xü, Huajian Zhu, Steven Wang *, Chao Yang *

\section{Theoretical Derivation}

The classic Lukas-Washburn equation was built on a Poiseulle flow. When the driving force is the capillary force only, i.e., pressure equals capillary force, and the channel is slitlike, the Hagen-Poiseulle relation is given as

$$
\frac{2}{d} \gamma \cos \theta=\frac{12}{d^{2}} \eta x \frac{\mathrm{d} x}{\mathrm{~d} t}
$$

where $x$ is the penetration height within the capillary, $t$ denotes time, $d$ is the width of the slit, $\gamma$ and $\theta$ indicate the surface tension and the contact angle respectively, and $\eta$ is the viscosity.

By integration, the time-dependent penetration height can be given as [1]

$$
x=\sqrt{\frac{\gamma d \cos \theta}{3 \eta}} \sqrt{t}
$$

When a slippage takes place in the flow, a slip length can be defined with the Navier boundary condition:

$$
v_{\text {slip }}=\left.\lambda_{s} \frac{\partial v_{x}}{\partial \mathrm{z}}\right|_{\mathrm{z}=0}
$$

Then Eq.(1) should be modified into

$$
\begin{gathered}
\frac{2}{d} \gamma \cos \theta=\frac{12}{\left(d^{2}+6 \lambda d\right)} \eta x \frac{\mathrm{d} x}{\mathrm{~d} t} \\
x=\sqrt{\left[\frac{(d+6 \lambda) \gamma \cos \theta}{3 \eta}\right] t}
\end{gathered}
$$

When we define a parameter $\mathrm{A}$ as

$$
A=\frac{6 \eta}{(d+6 \lambda) \gamma \cos \theta}
$$




\section{WILEY-VCH}

and calculate the velocity $\mathrm{d} x / \mathrm{d} t$ when $x$ equals to $L$ from Eq.(4), then we get the terminal velocity which is the imbibition velocity when the capillary is full with water:

$$
v_{\mathrm{T}}=\frac{1}{A L}
$$

In slits with sectional surface hydrophilicity, the governing equation with force balance like Eqs.(1) and (4) should be written as piecewise functions:

$$
\left\{\begin{array}{l}
\frac{2}{d} \gamma \cos \theta=\frac{12}{d^{2}} \eta x \frac{\mathrm{d} x}{\mathrm{~d} t} \quad x \leq L_{1} \\
\frac{1}{d} \gamma \cos \theta=\Delta P_{1}+\Delta P_{2}=\frac{3}{\left(d^{2}+3 \lambda_{1} d\right)} \eta_{1} L_{1} \frac{\mathrm{d} x}{\mathrm{~d} t}+\frac{3}{\left(d^{2}+3 \lambda_{2} d\right)} \eta_{2} x \frac{\mathrm{d} x}{\mathrm{~d} t} \quad L_{1}<x \leq L_{2}
\end{array}\right.
$$

And then the relationship between $x$ and $t$ can be given as

$$
\begin{cases}x=\sqrt{\frac{2}{A_{1}} \sqrt{t} \quad x \leq L_{1}} & \\ x=L_{1}+\frac{-A_{1} L_{1}+\sqrt{\left(A_{1} L_{1}\right)^{2}+2 A_{2} t}}{A_{2}} & L_{1}<x \leq L_{2}\end{cases}
$$

The condition of $A_{1}>A_{2}>0$ is required, otherwise the water will be detained at the spots of hydrophilicity difference. And from Eq.(9), we can get the terminal velocity for a sectional slit as

$$
v_{\mathrm{T}}=\frac{1}{A_{1} L_{1}+A_{2} L_{2}}
$$

and the condition of $A_{1}>A_{2}>0$ is similarly required.

[1] B. Henrich, C. Cupelli, M. Santer and M. Moseler, New J. Phys. 2008, 10, 113022. 